\title{
BMJ Open Study protocol for One Health data collections, analyses and intervention of the Sino-Swedish integrated multisectoral partnership for antibiotic resistance containment (IMPACT)
}

To cite: Sun Q, Wang Y, Hulth A, et al. Study protocol for One Health data collections, analyses and intervention of the SinoSwedish integrated multisectoral partnership for antibiotic resistance containment (IMPACT). BMJ Open 2018;8:e017832. doi:10.1136/ bmjopen-2017-017832

- Prepublication history and additional material for this paper are available online. To view these files, please visit the journal online (http://dx.doi. org/10.1136/bmjopen-2017017832).

SB and CSL contributed equally, $Q S$ and YW contributed equally.

Received 19 May 2017

Revised 2 November 2017 Accepted 6 November 2017

Check for updates

For numbered affiliations see end of article.

Correspondence to Dr Anette Hulth; anette.hulth@ folkhalsomyndigheten.se and Professor Yonghong Xiao; xia0-yonghong@163.com

\section{ABSTRACT}

Introduction To effectively minimise the emergence and dissemination of antibiotic resistant bacteria, a holistic One Health approach is called for. The Sino-Swedish Integrated Multisectoral Partnership for Antibiotic Resistance Containment is a cross-sectoral and integrated project on antibiotic resistance, conducted in Shandong Province in China. This paper outlines the overall study protocol for the project. To our knowledge, this is the first research programme aiming to take a true holistic approach across multiple sectors simultaneously in China, and the first to incorporate both antibiotic use and infection prevention and control in addition to antibiotic resistance patterns. The project aims to address gaps in current knowledge and seeks to improve the situation through a systemwide intervention. By using a One Health approach we can address important research questions that individual discipline investigations are unable to. The results obtained should thus more closely reflect the world in which human health, animal health and the environment are inextricably and intimately interlinked.

Methods and analysis Both quantitative and qualitative studies are included for households from 12 villages, their surrounding environment and a tertiary care hospital in a nearby town. The studies include analyses of antibiotic consumption for humans and pigs; qualitative and quantitative data on perceptions, knowledge and attitudes; faecal carriage of extended spectrum $\beta$-lactamase and carbapenemase-producing Enterobacteriaceae from pigs and humans, and occurrence in household drinking water, surface water, waste water and clinical bacterial isolates from the hospital. Carriage of methicillin-resistant Staphylococcus aureus in humans, household pigs and clinical bacterial isolates is also investigated. Furthermore, potential inter-relationships between these sources are analysed. A multifaceted One Health intervention is designed and implemented in 6 of the 12 villages. Repeated and continuous data collections take place over 2 years, where the repeated data collection is performed
Strengths and limitations of this study

- Antibiotic resistance is a complex global health challenge, therefore One Health approaches are needed to facilitate understanding and action.

- We incorporate both antibiotic use and infection prevention and control in addition to antibiotic resistance patterns from humans, pigs and their environment.

- The initial results from a baseline data collection in the community are used to design a contextually appropriate and sustainable One Health intervention package which aims to increase knowledge; improve antibiotic use; and indirectly limit the emergence and dissemination of antibiotic resistance.

- Residents might not remember or might respond in a way they think is socially desirable to the questions in the questionnaire, and they might not include all used antibiotics in the collection bags.

- The only animals included in the study are pigs.

after 1 year of intervention. Comparisons are made between intervention and control villages, before and after the intervention.

Ethics Ethics approval was obtained from the first Affiliated Hospital, College of Medicine, Zhejiang University, China, reference number 2015\#185 and 2015\#283.

\section{INTRODUCTION}

Antibiotics are used in both human and animal healthcare, with the result that antibiotics as well as resistant bacteria are emitted into the environment through manure and sewage. Resistant bacteria and resistance genes are continuously exchanged among the human, animal and environmental sectors. ${ }^{1-4}$ 
Antibiotic resistance is of utmost concern, as it is leading to rising morbidity, mortality and healthcare costs all around the world. ${ }^{56}$ To effectively minimise the emergence and dissemination of resistant bacteria, a holistic One Health approach is called for at both national and international levels, with collaborative actions in the fields of rational use of antibiotics and infection prevention and control, as recently emphasised by the United Nations' General Assembly. ${ }^{7}$ China plays a central role in such work, as it is one of the largest producers and consumers of antibiotics in the world. ${ }^{8}$ The problem of high prevalence of resistant bacteria among humans and animals has been extensively described in Chinese surveillance programmes and research studies. ${ }^{9-15}$ Similar resistance genotypes have been identified in humans and animals, ${ }^{16}$ and the same or related antibiotics are used in farm animals and humans. Additionally, information on the epidemiological background in rural China is lacking ${ }^{910} 17$ making it a suitable place for conducting these kinds of studies. In 2013, 10 research and governmental organisations in China and Sweden were granted funding from a joint call between the National Natural Science Foundation of China and the Swedish Research Council to pursue a project named Sino-Swedish Integrated Multisectoral Partnership for Antibiotic Resistance Containment, abbreviated IMPACT. ${ }^{18}$ Funding was granted for 5 years, and the project formally started in January 2014.

IMPACT is a cross-sectoral and integrated project on antibiotic consumption and resistance, conducted in China. The project includes both data collection and intervention components in the community in rural villages. The village residents commonly breed pigs and other animals in household backyard farms, and one village is close to a commercial pig farm. The project also includes a county tertiary hospital which serves a large area including the villages. The IMPACT project consists of four phases: (1) Joint problem formulation around a One Health approach to antibiotic resistance including development of shared and harmonised method protocols; (2) A baseline situation analysis, investigating the present situation of knowledge, attitudes, practices and perceptions on antibiotic use and antibiotic resistance across human, animal and environment sectors; (3) Design and implementation of a multifaceted context-specific intervention to prevent infections, improve antibiotic use in humans and animals, and limit the spread of resistant bacteria; (4) Evaluation of the intervention through a repeated data collection and situation analysis.

In this study protocol we present performed and coming data collections and the initial analyses of these data, as well as introduce a One Health intervention which is currently ongoing (as of May 2017). The studies were designed to meet the purpose of the IMPACT project, which is fourfold:

- to increase basic knowledge and understanding of the complex routes of dissemination of antibiotic resistance between different sectors (humans, animals and environment) by a cross-sectoral and bilateral approach
- to increase basic knowledge and understanding of factors influencing irrational use of antibiotics in humans and animals

- to integrate the resultant knowledge with existing evidence to design and pilot interventions aiming to limit development and spread of antibiotic resistance

- to promote adequate infection prevention and control and access to effective antibiotics for humans and animals for improved public and animal health, and consequently efficient, sustainable animal food production.

A few previous studies have aimed to link the prevalence of antibiotic resistance in different sectors such as human, animal, food and the environment. ${ }^{31920}$ To our knowledge, this is the first research programme aiming to take a true holistic approach across these sectors simultaneously in China, and the first to incorporate both antibiotic use and infection prevention and control in addition to antibiotic resistance patterns. The descriptive phase of the research programme, as detailed in this study protocol, will provide a thorough situation analysis with regard to the cross-sectoral epidemiology of resistant clones and genetic elements, as well as perceptions, knowledge, attitudes and practices related to antibiotic use. All instruments are developed in a close interdisciplinary and cross-sectoral collaboration, in line with the holistic thinking in the project. ${ }^{18}$

A data collection as described here is rarely found; we are collecting and characterising resistant bacteria at a household level, and in the same households we are simultaneously collecting information on knowledge, attitudes and practices related to antibiotic use. In addition we have information from nearby village clinics as well as from the environment surrounding the households. By using an interdisciplinary One Health approach we can address important research questions that individual discipline investigations are unable to, taking into account the biology of the bacteria as well as human practices and factors that influence them. The results obtained should thus more closely reflect the world in which human health, animal health and the environment are inextricably and intimately interlinked, and the effect of human practices in relation to the dissemination of antibiotic resistance. Furthermore, low-cost, scalable interventions are implemented and evaluated in a holistic manner, looking at antibiotic resistance patterns, knowledge, attitudes and perceptions and the possible interactions between them. Hence, the Sino-Swedish IMPACT project will contribute to the overall understanding of successful management of antibiotic resistance in a rural part of China, and will have broader implications far beyond this setting.

\section{METHODS AND ANALYSIS: DATA COLLECTION AND LABORATORY METHODS}

This is a mixed methods study with both continuous and repeated data collections over a 2-year period. The expected outcomes are both qualitative and quantitative. 
August 2016 -
July 2015
July 2017
August 2017
September 2017 -
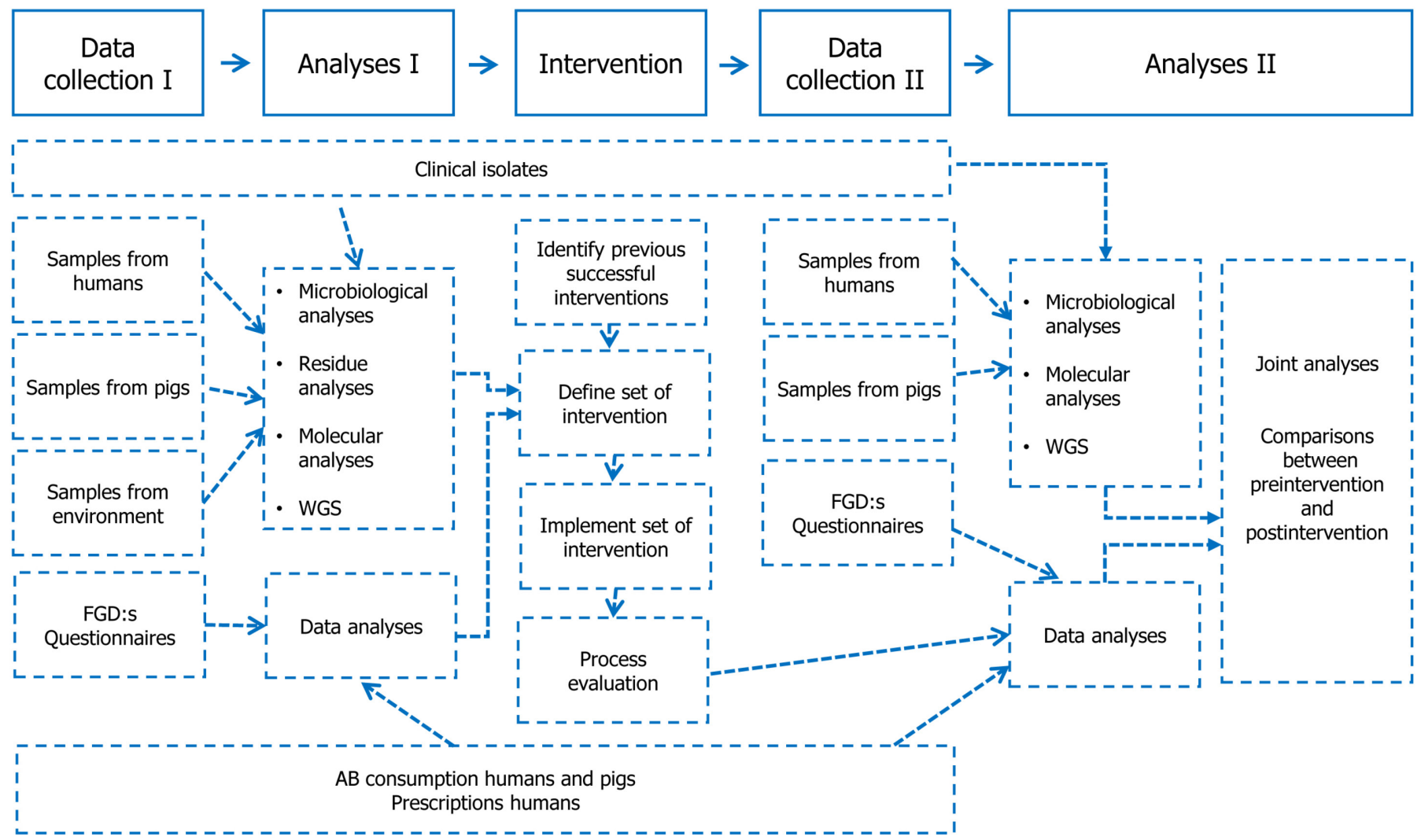

Figure 1 Main components of the community and hospital part of the Sino-Swedish Integrated Multisectoral Partnership for Antibiotic Resistance Containment (IMPACT) project and their relationships. FGDs, focus group discussions; WGS, whole genome sequencing.

Figure 1 outlines the main components of the community and hospital part of the IMPACT project as well as how they are related. We collect biological samples from humans (faecal and nasal) and pigs (faecal and skin) within households to identify prevalence and overlap of antibiotic resistant bacteria, focusing on extended spectrum $\beta$-lactamase (ESBL)-producing Escherichia coli and Klebsiella pneumoniae, carbapenemase-producing E. coli and K. pneumoniae, and methicillin-resistant Staphylococcus aureus (MRSA). Clinical isolates (eg, blood and urine) and environmental samples (eg, water and sediment) are also collected for the same purpose. ESBL-producing and carbapenemase-producing E. coli and K. pneumoniae of clinical origin are generally multidrug resistant. ${ }^{21}{ }^{22}$ Therefore the use of colistin has emerged as an important alternative in human medicine to treat these bacteria. ${ }^{23}$ However, recently transferable genes encoding colistin resistance, $m c r-1$ and $m c r-2$, were reported, ${ }^{124}$ which is why we also test all collected ESBL-producing and carbapenemase-producing $E$. coli and $K$. pneumoniae isolates for presences of these genes. We collect data on knowledge, attitudes and practices related to antibiotic use by interviewing village residents, village doctors and rural veterinarians. In addition, antibiotic use is measured in households, and prescription data are collected from village clinics. A repeated data collection, similar to the baseline data collection, takes place in the same villages after an intervention period. The intervention is designed to improve antibiotic use and limit the development and spread of antibiotic resistant bacteria.

\section{Setting and selection criteria}

The empirical data are collected in Shandong Province in eastern China. This is the second largest province in China by population and has slightly higher income and better health indicators than the average in China. The province has a population of 96 million in 17 cities and 140 counties, of which around half are rural. There were 1490 hospitals in 2013 with almost 340000 beds (974 general hospitals, 157 Chinese traditional hospitals and 347 special care hospitals). In addition there were 2308 community health centres and 1643 township health centres. ${ }^{25}$ In 2015 , the total production of meat was 12.5 million tons, including poultry meat (4.6 million tons), pork (3.8million tons) and beef (146000 tons) (statistics provided by Shandong Academy of Agricultural Sciences, personal communication).

We chose to conduct the research in a rural area because household pigs live in close proximity to humans and many residents work as farmers (ie, they have extended periods of contact with animals and the environment). ${ }^{2627}$ The particular area was chosen taking 
into account the number and scale of pig farms, the environment, the healthcare system (such as the size of the county hospital, the numbers of hospital beds, the professional level of the medical workers, etc), and conditions for performing research. The area has a population of 1.07 million, most of whom reside in the central city, and has a high middle level of economic development. The specific study site was selected based on the amount of families with backyard pigs, the distance to the location of the county government, the distribution of commercial farms and the cooperative degree of local township health centres.

\section{Selection of included villages}

We obtained basic information for all 73 villages in the selected area through the support of the local centre for disease prevention and control (CDC), including the number of residents, the number of households, the number of households with backyard pig farms and the distance to the township health centre. First, we selected all 17 villages containing at least 100 households. Among these 17 villages, we purposively included 12 villages in order to maximise the number of available backyard pig farms and village clinics. There are eight village clinics shared among the 12 villages. According to policies issued by the Ministry of Health, the main functions of the village clinics are to provide basic medical care services, to provide basic public health services including infectious disease control and chronic disease control, and to transfer patients to the higher level health institutions. The 12 villages were subsequently divided into six control villages and six intervention villages, by considering the number of households with and without pigs and the location of shared village clinics, to ensure a similar number of households with and without pigs as well as the same number of shared village clinics in the intervention and the control groups, respectively. Figure 2 shows a map of the location of the hospital and the control and intervention villages, respectively.

\section{Selection of households and participants}

Within the villages, the key unit of analysis is the household, and it is within each household that questionnaire data and microbiological samples from humans and pigs are collected.
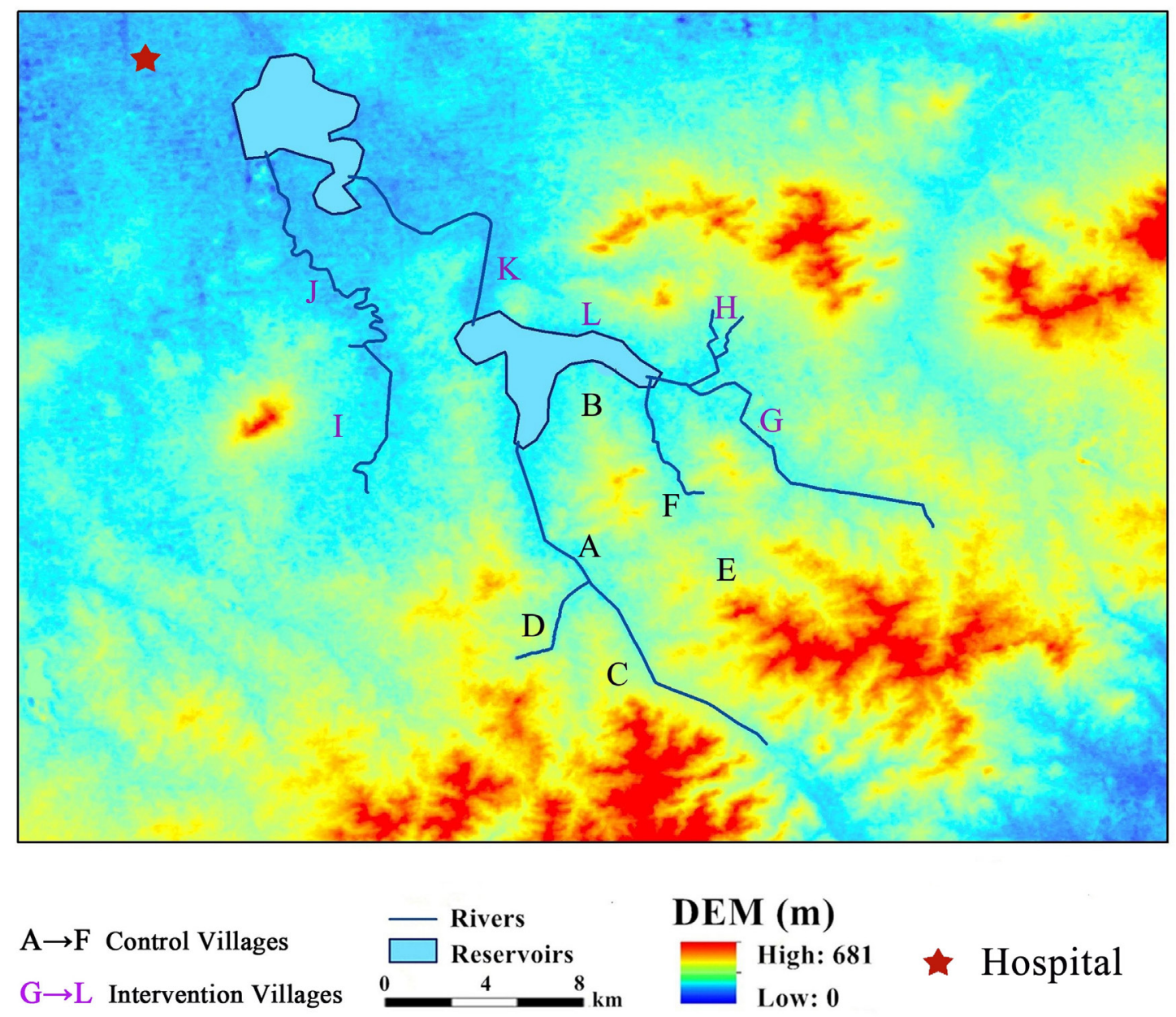

Figure 2 Map of the location of the hospital and the villages (control and intervention, respectively). DEM, digital elevation model. 
Based on the number of households in the selected villages and the feasibility of study, 65 households in each village were selected, using registers from the local CDC. Power calculations were made and analyses will be adjusted taking intracluster correlation into account. First, in each village between 5 and 35 households with backyard farms with at least one pig were randomly selected; the target was to include 35 households with backyard pig farms per village, but not all villages had this number of backyard farms. Households with large farms (defined as more than 50 pigs) were not eligible to participate, because these were considered to be 'specialist farms' rather than 'backyard farms'. Second, the remaining number of households needed to bring the total to 65 households in each village were selected using a matching sampling method based on the number of inhabitants in each household. Baseline sociodemographic data on the selected 780 households were obtained from local CDC registers, and were verified in the rural resident questionnaire. If the household declined participation at the day of the survey, or if no one was at home after two trials, a demographically similar household in the same village was selected to replace the omitted one.

Within each household, an adult resident ( $\geq 18$ years) was selected for participation in the microbiology sampling (one faecal sample and one nasal swab sample) and questionnaire study components. The selection was based on the participant's availability on the day of the study, and it prioritised the individual in the household who worked most closely with pigs (if relevant).

Within each household backyard pig farm, up to two faecal and two skin swab samples were taken from separate pens. The following sampling strategy was used:

- If there were two or more pens with weaners/ growers (age 70-100 days) or fatteners (age $>100$ days), two of these were selected. If possible, one with weaners/growers and one with fatteners were selected

- If there was only one pen with weaners/growers or fatteners, that pen and in addition, a pen with sows and piglets, or only a sow were selected

- If there were no pens with weaners/growers or fatteners, two pens with sows and piglets or only a sow were selected.

\section{Selection of a hospital serving the area}

The largest hospital in the main city in the area was selected for this study. It is a tertiary level B hospital (the hospital was a secondary level A hospital at the beginning of the project, and was upgraded just before the data collection began). At present (2017) there are 1700 beds and over 2100 staff. The hospital has 56 clinical departments and 7 departments of medical technology. In 2015 the outpatient department and emergency department of the hospital had over 600000 visits. The number of patients who underwent surgery was 16000 , and the number of patients discharged from the hospital was 70000 . The hospital was included to get additional information regarding resistance circulating in pathogens in the area.

\section{Training in microbiological methods}

A laboratory oriented workshop for those who would work with the microbiological analyses of the collected samples was organised before the baseline data collection started. The workshop was held in a microbiological laboratory in Shandong in February 2015 and contained both scientific theory and practice, and had participants from all sectors. Researchers and microbiologists from medicine and veterinary science were responsible for the teaching.

\section{Pilot study}

In June 2015 a pilot study was conducted in two villages within the study area to field test the data collection instruments and procedures and to train the research assistants. These villages are separate to the 12 selected villages (as described above), and so are not included in the study. Ten households were visited to test the questionnaire and the microbiology sampling procedures for humans and pigs. Furthermore, environmental samples were collected in both villages. Prescriptions were collected at one village clinic and the semistructured questionnaire was pilot tested with one village doctor. A feedback session was arranged immediately after the pilot day. Minor changes in the data collection procedures were made after the pilot study, including adjusting the sequence for the questionnaire survey and sample collection, and increasing the number of sampling teams.

\section{Baseline data collection from village residents}

\section{Focus group discussions (FGDs) with rural residents}

In total six FGDs with village residents were organised in February, April and June 2015, in six villages which are located in the study area but not selected for inclusion in the main components of the baseline study. An FGD question guide was developed based on previous experience ${ }^{28} 29$ and a literature review. Maximum variation sampling of adults was used to capture a wide range of viewpoints. In each FGD, 6 to 12 village residents were invited to participate, and among the six FGDs, three FGDs included residents who had backyard pig farms. All FGDs were conducted in Mandarin Chinese by locally trained research assistants and were recorded. The FGDs each lasted 45-70 min.

\section{Household data collection: logistics}

All selected households in the 12 villages were visited between 19 July and 26 July 2015. Forty research assistants were divided into two groups, with each group visiting one village per day. Each group was equipped with cool boxes, household lists, data labels, sampling material and protective clothing. Each group was then divided into eight subgroups ('household sampling teams') to collect data and samples at households within a village. Each household visit lasted between $20 \mathrm{~min}$ and $50 \mathrm{~min}$. 


\section{Household data collection: survey questionnaires}

A questionnaire was developed in English, translated into Mandarin Chinese, tested at the pilot in June 2015, revised and back-translated into English. The questionnaire is provided as an online supplementary file. Information from the two FGDs held in February 2015 (described above) was used to develop the household survey questionnaire.

The same person from which the microbiological samples (described below) were taken was invited to answer the questionnaire, in Mandarin Chinese. The questionnaire includes sections on participant and household sociodemographics, recent illnesses among household members and household pigs, and participants' knowledge, attitudes and practices concerning antibiotics for humans and pigs. Four questions on home storage of drugs for both human and pig use are included in the household survey questionnaire. The research assistant asked the participant to show their stored drugs and wrote down the names of all the drugs, then asked the participant to indicate which stored drugs they thought were antibiotics.

\section{Household data collection: sampling from humans and pigs for} microbiological analyses

All human and pig samples were collected using the ESwab collection and transport system (Copan, Brescia, Italy). All samples were marked with preprinted labels (described below). Disposable protective clothing, such as gloves, masks, shoe covers and disinfection were used to minimise risk of contamination or spread of pathogens between samples, pens, households and villages.

Sampling from humans: Fresh faecal samples were taken by the village residents themselves according to provided instructions and using ESwabs distributed the day before the household was visited by the household sampling team. The faecal samples were collected by the household sampling team on arrival at the household. Nasal swab samples were taken by a member of the household sampling team using ESwabs, and in general taken from the same person as the faecal sample. In case that person was unavailable, the nasal sample was taken from another person from the same household.

Sampling from pigs: In a pen, skin swab samples from up to three pigs were collected by rubbing the ESwab behind an ear of the pigs. Faecal samples were collected by dipping ESwabs in up to three fresh pig droppings.

\section{Collection of antibiotic consumption data in the households}

From May 2016 to July 2017, households' antibiotic consumption is being monitored in two control villages and two intervention villages. The data collection started immediately prior to the start of the intervention period (August 2016-July 2017). Within each village, a pragmatic selection of up to 25 households with backyard pig farms was included, and a further 25 households without backyard pig farms were selected by matching based on number of household residents. All included households had participated in the baseline study. Participating households maintain a weekly diary of illnesses and acute medications taken by household residents or given to pigs. The actual packages of given medications are collected in bags, separately for humans and pigs. The village doctor visits each participating household every fortnight to collect data and verify which medications have been used.

\section{Data collection from the village clinics}

\section{Collection of prescription data in the villages}

There are normally around 300 prescriptions per month per village clinic in the study area, and 50 prescriptions per month were selected using a random sampling method. From the eight village clinics, slightly more than 600 prescriptions per clinic covering the previous 12 months were collected at the time of baseline data collection (July 2015), then again prior to intervention (July 2016). This will be repeated a third time in July 2017, to cover a total of 3 years. The prescriptions are photographed at the clinics and relevant information is subsequently entered into a database (patient sociodemographics, diagnosis and details of medications prescribed). The prescription data analyses are stratified by clinic.

\section{Semistructured interviews with village doctors}

Semistructured interviews were conducted with all 19 village clinic doctors from the 8 village clinics associated with the 12 selected villages. An interview guide was developed based on previous experience ${ }^{28} 29$ and a literature review. All interviews were conducted in Mandarin Chinese by locally trained research assistants and were recorded. The interviews with the village doctors each lasted 40-60 min.

\section{Environmental sampling for microbiological and residue analyses}

Environmental samples were collected in the 12 villages during the same time period as when the baseline data were collected from the villages (July 2015). The samples collected consisted of different type of water (well, surface, tap and waste), sediment, manure, soil and cultured vegetables, when available. One litre of well water and 1 $\mathrm{L}$ surface water from rivers/streams were each collected in sterile glass bottles (Shuniu, China), brought to the local CDC laboratory and subjected to filtration through $0.45 \mu \mathrm{m}$ sterile membrane filters (Millipore, Billerica, USA). After the filtration, membranes were transferred into ESwabs and stored until analysis as described below. At least $30 \mathrm{~g}$ samples of river sediment and soil were collected in sterile plastic bottles and brought to the local laboratory for temporary storage. Vegetables were collected in plastic bags, transported to the local laboratory, and the surface was sampled using the ESwab collection and transport system, and stored until analysis as described below.

\section{Clinical isolates}

Clinical samples (blood, urine, wound secrete, ascites, puncture fluid and bile) are collected at the hospital 


\section{Household baseline data collection ( $=1$ )}

影响力IMPACT

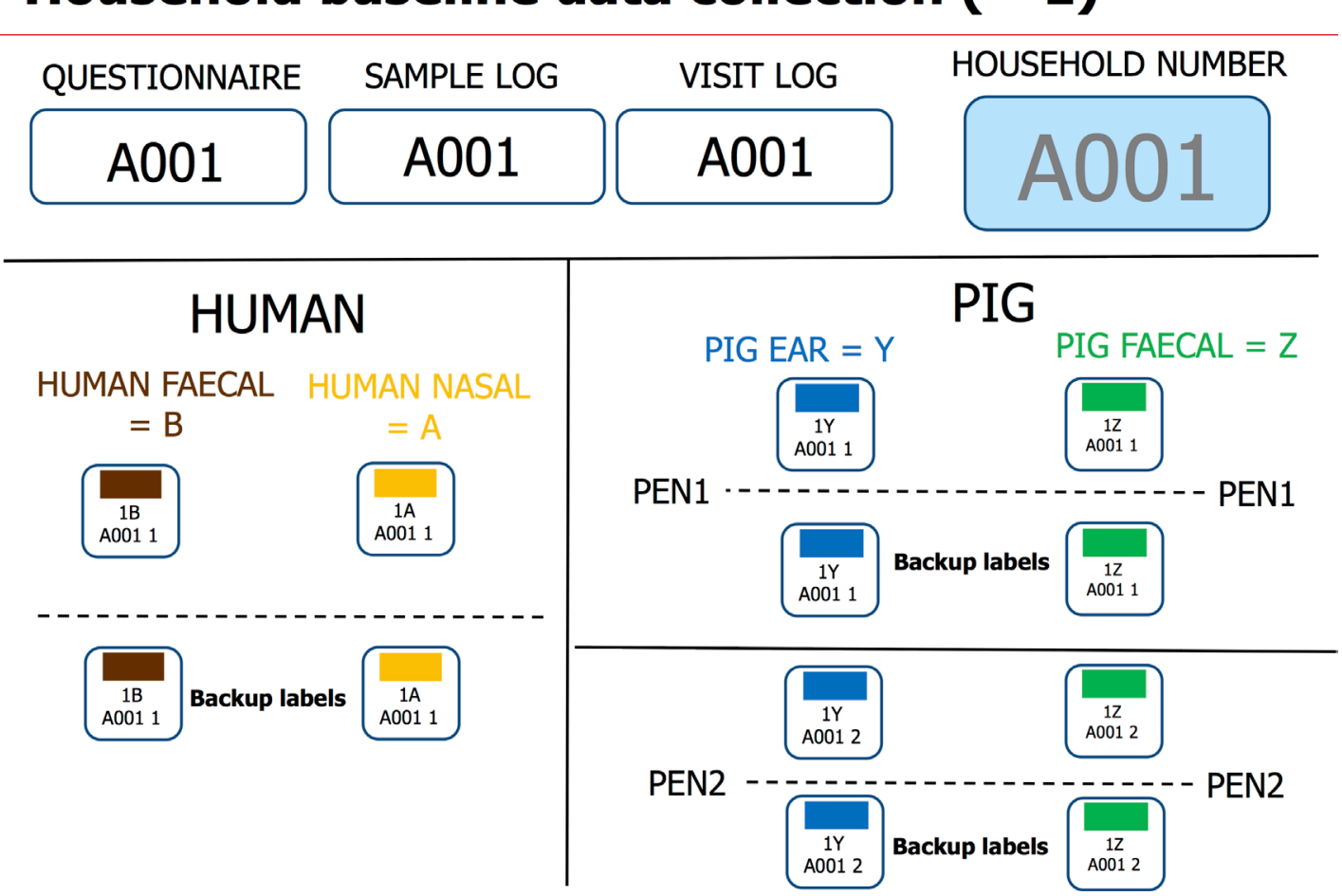

Figure 3 Sample label sheet. Labels for microbiological samples from humans and pigs as well as for the questionnaire from household 001 in village $A$. There is a spare label for each label type.

throughout the project period. Preliminary cultivation is performed at the hospital by streaking samples on Columbia blood agar plates (bioMérieux, Marcy l'Etoile, France). Suspected isolates of S. aureus, E. coli and $K$. pneumoniae are further identified at a university hospital laboratory.

\section{Data labelling}

A labelling system was designed for the community part of the IMPACT project to ensure that data are collected, stored and accessible in a way that permits cross-sectoral analyses. The labelling conveys time of collection (baseline or repeated collection); sample type (human nasal or faecal, pig skin or faecal); village code (A-L); household code (001-065); and, in the case of pig samples, number of pen (1 or 2$)$.

Labels were preprinted to avoid using hand-written labels, which can lead to errors of transcription and interpretation. For each household one sheet containing all labels for the household was prepared (see example in figure 3). The labels were colour coded to facilitate the labelling as well as future handling, with a unique colour given to human nasal, human faecal, pig skin and pig faecal samples, respectively. The labelling templates were developed in English and translated to Mandarin Chinese before being printed. The example in the figure is the English version of the sheet.

We tested the labelling system in the pilot and streamlined the process of sample collection for humans, pigs and the environment. We tested the labelling system again through a mock interview and sample collection (a simulation-based survey) in a training session before the formal baseline study. Before any labels were printed, the printing material was tested to ensure that the labels would stick and stay to the tubes when stored in low temperature freezers.

In addition to the preprinted labels, one sheet of paper per household, with the unique household identification (ID) label, was printed for keeping a log of the collected samples (see figure 4). Information was added to the sheet at the point of sampling, such as age and sex of the sampled person and pigs and breed of pig, and whether the faecal and nasal samples came from the same person. There were quality control questions about when the sample was taken and how it was stored before being collected.

We initially tried preassigning label IDs using the list of intended households, but this did not work because of necessary substitutions of households made on the day of the study. Therefore we reverted to assigning a specific label ID at the point of visiting individual households. The assigned code for each household was entered into an Excel spreadsheet for future reference at the end of each day of sampling.

\section{Laboratory methods baseline samples}

Microbiological samples were collected from around 740 households in the 12 villages in the baseline data collection, and approximately a third of these households had backyard pigs. 


\section{Sample label log: HUMAN}

Date samples collected: / 07 / 2015

Samples taken by:

\section{Human faeces swab sample}

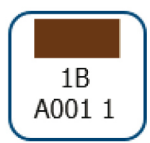

Number of samples:

Name:

Age:

YEARS OLD

Sex:

MALE/FEMALE

Daily pig contact?: $\quad Y E S / N O$

Date sample taken: YESTERDAY/TODAY/OTHER

Storage:

IN SHADE/NOT IN SHADE/OTHER

Was tube closed: FULLYCLOSED/NOT CLOSED

\section{Human nasal swab sample}

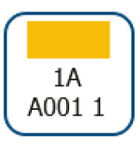

Number of samples:

Same person as faeces? YES/NO

(if NO, write information below)

Name:

Age:

YEARS OLD

Sex:

MALE/FEMALE

Daily pig contact?: $\quad Y E S / N O$
Transportation and storage of baseline samples

All samples from the villages were put in cool boxes with ice packs $\left(4-8^{\circ} \mathrm{C}\right)$ on collection. The environmental samples were kept in the cool boxes for between 1 hour and 3 hours, after which they were transported to the local CDC laboratory. The human and pig samples were kept in cool boxes throughout the day (up to 8 hours), and transported to the local CDC laboratory at the end of the day. At the local CDC, all samples were put into temporary storage for 1 day to 3 days at $4^{\circ} \mathrm{C}$. All samples were then transported by car (4hours) to Shandong CDC in cool boxes with ice packs. On arrival all samples were put in long-term storage at $-80^{\circ} \mathrm{C}$ until cultivation.

Clinical isolates are stored at $-80^{\circ} \mathrm{C}$ at the local hospital after sampling from the clinical cultures. Before transportation to the university hospital laboratory, isolates are streaked on agar plates and put in cool boxes with ice packs $\left(4-8^{\circ} \mathrm{C}\right)$. Clinical isolates are transported for analyses every second month, from July 2015.
Cultivation of baseline samples

Before cultivation, the samples are collected from the low temperature freezers and allowed to reach room temperature. Faecal, manure, waste water and outlet samples are cultivated for occurrence of presumptive ESBL-producing $E$. coli and $K$. pneumoniae by culturing the samples on ChromID ESBL agar (bioMérieux, Marcy l'Etoile, France). In parallel the samples are applied on ChromID CARBA agar (bioMérieux, Marcy l'Etoile, France) and ChromID OXA-48 agar (bioMérieux, Marcy l'Etoile, France) to identify presumptive carbapenem-resistant $E$. coli and $K$. pneumoniae. For water, soil, sediment and vegetable samples a pre-enrichment step in brain heart infusion broth is performed overnight before being applied on the plates. Each sample is also cultivated unselectively on a sheep blood agar plate (Babio, Jinan, China) to check the viability of the sample. All samples are applied on agar plates by a primary streak using the brush in the ESwab and then substreaked using a sterile $1 \mu l$ plastic 
loop. The agar plates are incubated for 18-24hours at $35^{\circ} \mathrm{C}$. After incubation, growth is recorded and suspected E. coli and K. pneumoniae colonies are identified based on colour and morphology, according to the manufacturer's instructions (bioMérieux, Marcy l'Etoile, France), subcultured on CHROMagar Orientation agar (CHROMagar Company, Paris, France) and incubated overnight at $35^{\circ} \mathrm{C}$. From the CHROMagar Orientation agar suspected E. coli or K. pneumoniae are transferred to storage tubes with glycerol and stored in $-70^{\circ} \mathrm{C} /-80^{\circ} \mathrm{C}$ until further analyses.

Nasal samples from humans and skin samples from pigs are pre-enriched by transferring $200 \mu \mathrm{l}$ of ESwab fluid to $1.3 \mathrm{~mL} 7.5 \%$ sodium chloride broth (Beijing Land bridge Technology, Beijing, China) with $75 \mathrm{mg} / \mathrm{L}$ aztreonam. Pre-enrichment is performed in closed $1.5 \mathrm{~mL}$ Eppendorf tubes overnight at $35^{\circ} \mathrm{C}$. From the pre-enrichment, $10 \mu \mathrm{l}$ is transferred to a CHROMagar MRSA plate (CHROMagar Company, Paris, France) and incubated at $35^{\circ} \mathrm{C}$ for 18-24 hours. After this incubation, growth on CHROMagar MRSA is recorded and these suspected MRSA colonies are subcultured on Baird-Parker (BP) agar (Beijing Land Bridge Technology, Beijing, China) overnight at $35^{\circ} \mathrm{C}$. From the BP agar, suspected MRSA colonies are transferred to storage tubes with glycerol and stored in $-70^{\circ} \mathrm{C} /-80^{\circ} \mathrm{C}$ until further analyses.

After applying samples on the respective agar plates, the remaining fluid in the ESwab tubes is transferred to $2 \mathrm{~mL}$ tubes and mixed with $30 \%$ glycerol for long-term storage at $-70^{\circ} \mathrm{C} /-80^{\circ} \mathrm{C}$.

Clinical samples are cultivated by BacT/ALERT 3D system (bioMérieux, Marcy l'Etoile, France) or on blood agar plates (bioMérieux, Marcy l'Etoile, France), $18-24$ hours at $37^{\circ} \mathrm{C}$, and MRSA isolates are identified by the VITEK-2 system (bioMérieux, Marcy l'Etoile, France).

\section{Verification of MRSA and ESBL-producing and carbapenemase-}

\section{producing $E$. coli and $K$. pneumoniae isolates}

Species confirmation of presumptive E. coli and K. pneumoniae isolates is performed by matrix-assisted laser desorption ionisation-time of flight mass spectrometry (MALDI-TOF-MS). For isolates of environmental and human origin, Shimadzu with saramis premium (Shimadzu Corporation, Kyoto, Japan) is used, whereas Bruker Daltonik GmbH (Bremen, Germany) is used for isolates of pig origin.

Species identification for clinical isolates is performed using VITEK-2 system (bioMérieux, Marcy l'Etoile, France) and Bruker MALDI-TOF-MS (Bruker, Germany). ESBL production is confirmed by the double disc diffusion method, using cefotaxime, ceftazidime, each alone and in combination with clavulanic acid according to the manufacturer's protocol (Oxoid, Basingstoke, UK). E. coli ATCC 25922 and K. pneumoniae ATCC 700603 are used as control strains.

Verified ESBL-producing E. coli and K. pneumoniae isolates are checked for presence of genes encoding ESBL $\left(b l a_{\mathrm{CTX}-\mathrm{M}} b l a_{\mathrm{SHV}}\right.$ and $\left.b l a_{\mathrm{TEM}}\right)$, while resistant isolates showing no inhibition with clavulanic acid are also checked for genes encoding pAmpC $\left(b l a_{\mathrm{CIT}}, b l a_{\mathrm{MOX}}, b l a_{\mathrm{FOX}}, b l a_{\mathrm{ACC}}\right.$ $\left.b l a_{\mathrm{DHA}}, b l a_{\mathrm{EBC}}\right)$ by conventional PCR or real-time PCR. ${ }^{30} 31$ Suspected carbapenemase-producing E. coli and K. pneumoniae are tested for carbapenemase production using the CNPt-direct ${ }^{32}$ and for presence of the genes encoding NDM, KPC, VIM, IMP and OXA-48 by PCR. ${ }^{33}$

Suspected MRSA isolates are confirmed with PCR by detecting spa or nuc, mecA, mecC and PVL. ${ }^{34} 35$

\section{Testing for genes encoding transferable colistin resistance}

All verified ESBL-producing and/or carbapenemase-producing E. coli and K. pneumoniae are tested for presence of the gene $m c r-1$ using $\mathrm{PCR}^{1}$ and if negative for $m c r-1$ they are also checked for presence of $m c r-2 .{ }^{24}$ PCR products are sequenced using Sanger sequencing at Macrogen (Seoul, South Korea), BGI (Beijing, China) or Biosune Biotechnology (Shanghai, China).

\section{Antibiotic susceptibility testing}

The confirmed MRSA and the ESBL-producing and carbapenemase-producing isolates are cultured on blood agar, incubated overnight at $35^{\circ} \mathrm{C}$, and then subjected to antibiotic susceptibility testing using agar dilution with Müller-Hinton agar (Oxoid), according to the Clinical and Laboratory Standards Institute (CLSI) document M100-S25 (2015). Susceptibility is assessed using European Committee on Antimicrobial Susceptibility Testing (EUCAST) clinical break points and epidemiological cut-off values, ${ }^{36}$ or CLSI break points ${ }^{37}$ are used when EUCAST is unavailable.

Antibiotic panel for $\mathrm{K}$. pneumoniae and $\mathrm{E}$. coli isolates: Amikacin (Zhejiang Conba Biological Pharmaceutical). Amoxicillin-clavulanic acid and cefotaxime (National Institute for the Control of Pharmaceutical and Biological Products). Cefoxitin, ceftazidime, meropenem, piperacillin/tazobactam, tigecycline and colistin (Beijing Ouhe technology). Ciprofloxacin (Shandong Jintaiyang Pharmaceutical). Florfenicol (Zhejiang Hisoar Pharmaceutical). Fosfomycin (SIGMA-ALDRICH). Gentamicin (Aladdin Industrial Corporation). Nitrofurantoin (J\&K Scientific). Tetracycline (National Institute for the Control of Pharmaceutical and Biological Products). Trimethoprim/sulfa (Shandong Xinhua Pharmaceutical).

Antibiotic panel for MRSA: Cefoxitin, ciprofloxacin, clindamycin, florfenicol, fusidic acid, gentamicin, linezolid, streptomycin, vancomycin, tigecycline and trimetoprim/ sulfa (Beijing Ouhe technology). Erythromycin (TCI (Shanghai) Chemical Industry Development). Rifampicin (J\&K Scientific). Tetracycline, tiamulin (Shandong Shengli). Virginiamycin (Chemsky (Shanghai) International).

\section{Antibiotic residue analysis}

High-performance liquid chromatography-tandem mass spectrometry (HPLC-MS/MS) is used for detection and determination of the concentration of antibiotic residues in the environmental samples. 
Reagents and chemicals: Antibiotics (metronidazole, sulfapyridine, norfloxacin, levofloxacin, ciprofloxacin, enrofloxacin, doxycycline, sulfamethoxazole, florfenicol and chloramphenicol) for preparation of standard solutions were purchased from Sigma-Aldrich (St Louis, Missouri, USA). Methanol and acetonitrile (HPLC grade) were obtained from Fisher (New Jersey, USA). Oxalic acid, phosphorous acid, disodium hydrogen phosphate $\left(\mathrm{Na}_{2} \mathrm{HPO}_{4}\right)$, and EDTA disodium salt $\left(\mathrm{Na}_{2} \mathrm{EDTA}\right)$ were used as analytical reagents (Beijing Reagent Company). Stock solutions of the standards were prepared in methanol at a concentration of $100 \mathrm{mg} / \mathrm{L}$ and stored at $-18^{\circ} \mathrm{C}$. Working standard solutions at a concentration of $10 \mathrm{mg} / \mathrm{L}$ were diluted from the stock solutions before use. Working mixed standard solutions were prepared by mixing the above solutions and diluting with methanol.

Sampling preparation: Soil and sediment samples are freeze-dried and sieved through a $0.5 \mathrm{~mm}$ pore size. For extraction, $3.0 \mathrm{~g}$ soil is mixed with $13 \mathrm{~g}$ EDTA washed diatomite in a mortar which is put into a $34 \mathrm{~mL}$ extraction cell. Dionex glass fibre filters are placed at the bottom and top of the extraction cell to avoid the obstruction of the end caps by the soil and diatomite particles. The analytes are extracted with methanol-citric acid $(\mathrm{pH}=4.7)$ at $70^{\circ} \mathrm{C}$ and 1500 psi for $10 \mathrm{~min}$ of static time, in one cycle, at $60 \%$ of flush volume, purged for $60 \mathrm{~s}$ with nitrogen. The accelerated solvent extraction (ASE), containing substantial amounts of methanol, is evaporated to a half volume, then diluted with water to a methanol content below $5 \%$. SAX-HLB tandem cartridges are preconditioned with $5 \mathrm{~mL}$ methanol and $5 \mathrm{~mL}$ water. The ASE extracts are passed through tandem cartridges at a speed of $3 \mathrm{~mL} /$ min. Subsequently, the HLB is washed with $10 \mathrm{~mL}$ water to flush impurity and freeze-dried for $15 \mathrm{~min}$. After that, the HLB cartridge is eluted with $6 \mathrm{~mL}$ methanol. Eluant is evaporated with nitrogen at $40^{\circ} \mathrm{C}$ and reconstituted with $1 \mathrm{~mL}$ methanol-water $(50: 50, \mathrm{v} / \mathrm{v})$, and passed through $0.22 \mu \mathrm{m}$ organic membrane filter before analysis.

Water samples preparation: SAX-HLB tandem cartridges are preconditioned with $5 \mathrm{~mL}$ methanol and $5 \mathrm{~mL}$ water. Water samples are passed through tandem cartridges at a speed of ca $3 \mathrm{~mL} / \mathrm{min}$, after which cartridges are washed with $10 \mathrm{~mL}$ water and freeze dried for $15 \mathrm{~min}$. The cartridges are then eluted with $6 \mathrm{~mL}$ methanol and eluants are evaporated with nitrogen at $40^{\circ} \mathrm{C}$ and reconstituted in $1 \mathrm{~mL}$ methanol-water $(50: 50, \mathrm{v} / \mathrm{v})$. Eluents are then passed through $0.22 \mu \mathrm{m}$ organic membrane filter.

Vegetable samples preparation: Lyophilised vegetables $(1.0 \mathrm{~g})$ are mixed with $10 \mathrm{~mL}$ of acetonitrile and hydrochloric acid (125:1, v:v) and homogenised with ultrasonication for $15 \mathrm{~min}$. The homogenate is then centrifuged at $4000 \times \mathrm{g}$ in air cooled conditions of $4^{\circ} \mathrm{C}$ for $10 \mathrm{~min}$ and the supernatants are collected in amber coloured bottles. The extraction process is performed three times with fresh acid acetonitrile. The three/four supernatants are mixed in a brown bottle and are liquid-liquid extracted with $20 \mathrm{~mL}$ of hexane. The extraction process using HLB cartridges is identical to soil extraction.
HPLC-MS/MS analysis: Separation of antibiotics is carried out using an XDB-C18 column $(4.6 \times 50 \mathrm{~mm}$, $1.8 \mu \mathrm{m}) ; 10 \mu \mathrm{L}$ of the dissolved extract is injected into the chromatographic system. The chromatographic mobile phase consists of $0.1 \%$ formic acid (A) and acetonitrile (B). The mobile phase gradient is ramped at a flow rate of $0.4 \mathrm{~mL} / \mathrm{min}$ from $10 \%$ B to $40 \%$ in $6 \mathrm{~min}$ and $40 \%$ to $95 \%$ in $2 \mathrm{~min}$, kept for $2 \mathrm{~min}$, then ramped to $10 \%$ in $5 \mathrm{~min}$. The column temperature is set to $20^{\circ} \mathrm{C}$. The nebuliser pressure is set to $30 \mathrm{psi}$, and the drying gas is $\mathrm{N}_{2}$. The flow rate and temperature of drying gas is $10 \mathrm{~L} / \mathrm{min}$ and $350^{\circ} \mathrm{C}$. The capillary voltages are $3000 \mathrm{~V}$.

\section{METHODS AND ANALYSIS: COMMUNITY INTERVENTION AND REPEATED DATA COLLECTION \\ One Health intervention}

The initial results from the baseline data collection in the community were used to design a contextually appropriate and sustainable One Health intervention package which aims to increase knowledge; improve antibiotic use; and indirectly limit the emergence and dissemination of antibiotic resistance. The intervention package is implemented in six of the 12 villages from August 2016 to July 2017, with the remaining six villages serving as controls for the forthcoming evaluation of the intervention. No intervention is conducted in the hospital from which samples are collected as part of the project.

The intervention consists of training for village doctors and veterinarians; for village residents, there are lectures, educational posters, booklets and educational audio tapes. The training for doctors and veterinarians aims to promote rational prescribing of antibiotics. We designed training according to the local village doctors' and veterinarians' education level, and the main contents include knowledge on rational antibiotic use (indication, usage and dosage, and how to provide health education to patients). The lectures for village residents (including people both with and without backyard pigs) consist of information on antibiotics and drug resistance, which aims to improve their knowledge on rational antibiotics use. These lectures take place at four occasions during the 12-month period. The village residents who participate in lectures also receive a booklet regarding the definition of antibiotics, antibiotic use indications, harmful consequences of irrational antibiotic use and how to use antibiotics rationally. We place posters around intersections in the villages or by residents' gathering places, such as the door of the supermarket or village clinic, or outer wall of a house located on the main road. The posters include a brief introduction to the immune system and how to deal with upper respiratory tract infections such as common cold. In addition, audio tapes are broadcast in each village once in the morning and once in the evening, every Tuesday, Thursday and Saturday. The audio tape content is mainly concerned with knowledge on antibiotics and aspects about antibiotic use and antibiotic resistance in humans, in particular in children, animals and environment. 
The intervention package was designed in close cooperation with the local CDC, the county hospital, the township health centre, and village doctors and veterinarians. The township health centre and village doctors assist by arranging lectures, putting up posters, distributing booklets and broadcasting the audio tapes.

The implementation process is followed closely by monitoring the number of participants, number of booklets and number of posters distributed in each village. A process evaluation form is completed by the researchers from Shandong University when they monitor the intervention with local partners. After the training sessions, the researchers interview the village doctors to collect their reflections on the content and to understand if further support is needed. For the village residents, 15 village residents are selected randomly from every intervention village and are given a questionnaire to collect their reflections on the lecture, the posters and the broadcasting as well as the booklet.

\section{Repeated data collection}

A second data collection is carried out in all 12 villages, using the same instruments and following the same methods as for the baseline data collection described in this protocol. This collection takes place in August 2017, after the intervention period has finished. During this second data collection, the same households as during the first data collection are visited. The same questions are posed, and the same kind of samples are collected from the same individuals as during the baseline data collection. (If the person sampled for the baseline is unavailable, we define the household as 'lost for follow-up', even if other household members are available for the repeated data collection.) Also the same microbiological and molecular methods are used. The unique ID enables linking the data between the two data collection occasions.

\section{METHODS AND ANALYSIS: DATA MANAGEMENT AND ANALYSES Data management}

Data files are prepared according to the study design and outcome variables, with particular consideration to joint analyses. As described earlier, unique identifiers are used for sample tracing from the community to the laboratory and also to determine the sample flow within the laboratory. A team of senior researchers supervise data entry. Data entry is carefully followed and quality assured through repeated random checks. Special arrangements are made to maintain confidentiality. For qualitative studies (FGDs and interviews), the recorded material is stored in a secured location. Any identity of the interviewee is removed from the transcripts before analysis.

\section{Data analyses}

Data analyses for epidemiological data

The questionnaires are cross-checked on the day of completion. Data are double-entered into an Access database, cleaned and translated. Both descriptive and inferential statistics are used to analyse the data, including making comparisons between villages and between households with and without backyard farms for outcomes such as: knowledge of good antibiotic use practices, awareness of antibiotic resistance, household storage of antibiotics. Isolates are characterised using both phenotypical and genotypical methods in order to elucidate relatedness and epidemiology of isolates within and between humans, pigs and the environment.

Comparisons will be performed using $\mathrm{X}^{2}$ tests/Student's $t$-test depending on the variable type. Furthermore, we will study the differences in the outcome variables using appropriate statistical methods, such as regression models and time series analyses. When relevant, clustering will be taken into account in the analyses.

The quantitative data from the household survey questionnaires will be analysed using STATA (StataCorp College Station, Texas, USA) or SPSS V.17.0 (SPSS Inc, Chicago, Illinois, USA).

\section{Analyses of prescription data}

Prescription data are double-entered into an Access database, cleaned and translated into English. Antibiotics for systemic use is coded using the Anatomical Therapeutic Chemical Classification System, ${ }^{38}$ and attempts will be made to code diagnoses according to the International Statistical Classification of Diseases and Related Health Problems, 10th revision (ICD-10). Summary statistics will be used to describe the patterns of antibiotic use in the village clinics. Proportions of prescriptions for acute respiratory tract infections containing antibiotics will be calculated, as well as proportions of prescriptions containing multiple antibiotics.

\section{Combined quantitative analyses}

All quantitative data will be linked and analysed in a combined manner to get an overall 'One Health picture' where associations between resistance from different sources (humans, pigs and environment), prescribing patterns and data from the survey questionnaires may be discovered.

\section{Analyses of FGDs and semistructured interviews}

The transcripts of FGDs and the semistructured interviews were translated into English, and are analysed using manifest and latent content analysis with side-by-side comparison of English and original Chinese transcripts. ${ }^{39} 40$

\section{Evaluation of the intervention}

Data collected by all instruments will be compared before and after the intervention; the impacts of the intervention will be evaluated using the difference in differences method, and between intervention and control villages using suitable regression models adjusted for clustering effects. The intervention implementation process will also be evaluated.

Due to the fact that in China the awareness of the problems with antimicrobial resistance is increasing among the policy makers, other interventions from, for example, 
local or national authorities may influence the situation and should therefore be included during analyses. An example of such an event is the ban of colistin as feed additive in farm animal in April 2017. Collaboration with local CDC and local veterinarians as well as partners from provincial and national levels ensures that information of such events is considered.

\section{ETHICS AND DISSEMINATION}

Before any type of data collection began, oral and written information was provided in the local language, Mandarin Chinese. All village doctors and participants from households signed consent forms before answering survey questions. All participants were informed that they could withdraw at any point and that confidentiality would be guaranteed. If a participant was unable to write their name, the research assistant signed on behalf of the participant. Permission was obtained for audio recording interviews and FGDs. Consent will be taken again before the repeated data collection.

All investigators will have access to the final data sets. The results will be submitted to peer-reviewed journals for publication as well as presented at national and international conferences. Informing policy makers at all levels is a crucial aspect of the dissemination and will be done from local to international levels. Publications are anticipated from 2018 and onwards, while policy makers are informed throughout the project period. During the intervention period, data from the preintervention data collection were provided in a customised way to the target groups. The residents as well as local health providers and policy makers will be provided with results at an aggregated level after the study has ended.

\section{Author affiliations}

${ }^{1}$ Shandong University, Center for Health Management and Policy, Jinan, China ${ }^{2}$ NHFPC (Shandong University), Key Laboratory of Health Economics and Policy Research, Jinan, China

${ }^{3}$ China Agricultural University, College of Veterinary Medicine, Beijing, China

${ }^{4}$ Public Health Agency of Sweden, Solna, Sweden

${ }^{5}$ Department of Public Health Sciences, Global Health - Health Systems and Policy, Karolinska Institutet, Solna, Sweden

${ }^{6}$ First Affiliated Hospital, College of Medicine, Zhejiang University, Hangzhou, China

${ }^{7}$ Department of Clinical and Experimental Medicine, Linkopings universitet,

Linkoping, Sweden

${ }^{8}$ Department of Environment and Health, School of Public Health, Shandong University, Jinan, China

${ }^{9}$ Shandong Center for Disease Control and Prevention, Jinan, Shandong, China

${ }^{10}$ Shandong Provincial Key Laboratory of Infectious Disease Control and Prevention, Jinan, China

${ }^{11}$ Shandong Academy of Agricultural Sciences, Jinan, China

${ }^{12}$ Key Laboratory of Animal Epidemic Prevention and Breeding in Shandong Province, Jinan, China

${ }^{13}$ Hebei Provincial Center for Disease Control and Prevention, Shijiazhuang, China

${ }^{14}$ Center for Health Management and Policy, Shandong University, Jinan, Shandong, China

${ }^{15}$ Affiliated Hospital of Nanjing University Medical School, Nanjing Drum Tower Hospital, Jiangsu, China

${ }^{16}$ Risk and Benefit Assessment Department, National Food Agency, Uppsala, Sweden

${ }^{17}$ National Veterinary Institute, Uppsala, Sweden
Collaborators Otto Cars, Björn Berglund, Zhenqiang Bi, Christina Greko, Gaetano Marrone, Jianzhong Shen, Göran Tomson and Maria Tärnberg.

Contributors All authors were involved in the design of the study and the development of instruments. QS, YW, XL, ZB, YLi, YLu, CS, YZ, BZ, BC, PS, LD and OJD participated in the data collection. QS, YW, YX, LEN, ZB, YLi, HY, YLu, MN, CS, $Y Z, B Z, B C, P S, L D, X X, 0 J D, S B$ and CSL participated in the initial analyses. QS, AH, LEN, XL, HY, MN, CS, BZ, OJD, SB and CSL drafted the manuscript and QS, YW, AH, YX, LEN, YLi, HY, MN, CS, BC, LD, XX, JO, SL, OJD, SB and CSL gave critical input. All authors read and approved the final manuscript.

Funding This study is part of the Sino-Swedish IMPACT project which is funded by the Swedish Research Council (grant D0879801) and National Natural Science Foundation of China (grant 81361138021).

Competing interests None declared.

Patient consent Obtained.

Ethics approval First Affiliated Hospital, College of Medicine, Zhejiang University, China, reference number 2015 no. 185 and 2015 no.283.

Provenance and peer review Not commissioned; externally peer reviewed.

Data sharing statement The data sets generated and analysed during the current study are available on reasonable request from the corresponding author.

Open Access This is an Open Access article distributed in accordance with the Creative Commons Attribution Non Commercial (CC BY-NC 4.0) license, which permits others to distribute, remix, adapt, build upon this work non-commercially, and license their derivative works on different terms, provided the original work is properly cited and the use is non-commercial. See: http://creativecommons.org/ licenses/by-nc/4.0/

(C) Article author(s) (or their employer(s) unless otherwise stated in the text of the article) 2018. All rights reserved. No commercial use is permitted unless otherwise expressly granted.

\section{REFERENCES}

1. Liu YY, Wang Y, Walsh TR, et al. Emergence of plasmid-mediated colistin resistance mechanism MCR-1 in animals and human beings in China: a microbiological and molecular biological study. Lancet Infect Dis 2016;16:161-8.

2. Keen PL, Montforts M. Antimicrobial resistance in the environment: Wiley-Blackwell, 2012.

3. Catry B, Van Duijkeren E, Pomba MC, et al. Reflection paper on MRSA in food-producing and companion animals: epidemiology and control options for human and animal health. Epidemiol Infect 2010;138:626-44.

4. Diwan V, Tamhankar AJ, Khandal RK, et al. Antibiotics and antibioticresistant bacteria in waters associated with a hospital in Ujjain, India. BMC Public Health 2010;10:414.

5. Global action plan on antimicrobial resistance. http://apps.who. int/iris/bitstream/10665/193736/1/9789241509763_eng.pdf?ua=1 (accessed 18 May 2017).

6. World Health Organisation. The evolving threat of antimicrobial resistance - Options for action 2012. http://www.who.int/ patientsafety/implementation/amr/publication/en/ (accessed 18 May 2017).

7. Resolution adopted by the general assembly on 5 October 2016 : $71 / 3$. Political declaration of the high-level meeting of the general assembly on antimicrobial resistance. http://www.un.org/en/ga/ search/view_doc.asp?symbol=A/RES/71/3 (accessed 18 May 2017).

8. Zhang QQ, Ying GG, Pan CG, et al. Comprehensive evaluation of antibiotics emission and fate in the river basins of China: source analysis, multimedia modeling, and linkage to bacterial resistance. Environ Sci Technol 2015;49:6772-82.

9. Sun P, Bi Z, Nilsson M, et al. Occurrence of blaKPC-2, blaCTX-M, and $\mathrm{mcr}-1$ in enterobacteriaceae from well water in rural China. Antimicrob Agents Chemother 2017;61.

10. Sun Q, Tärnberg M, Zhao L, et al. Varying high levels of faecal carriage of extended-spectrum beta-lactamase producing Enterobacteriaceae in rural villages in Shandong, China: implications for global health. PLoS One 2014;9:e113121.

11. Zhu YG, Johnson TA, Su JQ, et al. Diverse and abundant antibiotic resistance genes in Chinese swine farms. Proc Natl Acad Sci U S A 2013;110:3435-40.

12. Yezli S, Li H. Antibiotic resistance amongst healthcare-associated pathogens in China. Int J Antimicrob Agents 2012;40:389-97. 
13. Xiao YH, Giske CG, Wei ZQ, et al. Epidemiology and characteristics of antimicrobial resistance in China. Drug Resist Updat 2011;14:236-50.

14. Chen X, Naren GW, Wu CM, et al. Prevalence and antimicrobial resistance of Campylobacter isolates in broilers from China. Vet Microbiol 2010;144:133-9.

15. Heddini A, Cars O, Qiang S, et al. Antibiotic resistance in China--a major future challenge. Lancet 2009;373:30.

16. Wang Y, Lv Y, Cai J, et al. A novel gene, optrA, that confers transferable resistance to oxazolidinones and phenicols and its presence in Enterococcus faecalis and Enterococcus faecium of human and animal origin. J Antimicrob Chemother 2015;70:2182-90.

17. Bi Z, Berglund B, Sun Q, et al. Prevalence of the mcr-1 colistin resistance gene in extended-spectrum $\beta$-lactamase-producing Escherichia coli from human faecal samples collected in 2012 in rural villages in Shandong Province, China. Int J Antimicrob Agents 2017;49:493-7.

18. Cars O, Xiao Y, Stålsby Lundborg C, et al. Building bridges to operationalise one health - A Sino-Swedish collaboration to tackle antibiotic resistance. One Health 2016;2:139-43.

19. Boonyasiri A, Tangkoskul T, Seenama C, et al. Prevalence of antibiotic resistant bacteria in healthy adults, foods, food animals, and the environment in selected areas in Thailand. Pathog Glob Health 2014;108:235-45.

20. Scientific opinion on the public health risks of bacterial strains producing extended-spectrum $\beta$-lactamases and/or ampc $\beta$-lactamases in food and food-producing animals. EFSA $J$ 2011:9:2322.

21. Naseer U, Sundsfjord A. The CTX-M conundrum: dissemination of plasmids and Escherichia coli clones. Microb Drug Resist 2011;17:83-97.

22. Poirel L, Pitout JD, Nordmann P. Carbapenemases: molecular diversity and clinical consequences. Future Microbiol 2007;2:501-12.

23. Wang $Y$, Tian GB, Zhang R, et al. Prevalence, risk factors, outcomes, and molecular epidemiology of $m c r-1$-positive Enterobacteriaceae in patients and healthy adults from China: an epidemiological and clinical study. Lancet Infect Dis 2017;17:390-9.

24. Xavier BB, Lammens $C$, Ruhal R, et al. Identification of a novel plasmid-mediated colistin-resistance gene, mcr-2, in Escherichia coli, Belgium, June 2016. Eurosurveillance 2016;21:27.

25. Shandong health development report in 2013 (in Chinese). htt p://www.sdwsjs.gov.cn/tjsj_763/zytjbg/201407/P0201501305 97230822446.pdf (accessed 18 May 2017).
26. Schmithausen RM, Schulze-Geisthoevel SV, Stemmer F, et al. Analysis of transmission of MRSA and ESBL-E among pigs and farm personnel. PLoS One 2015;10:e0138173.

27. Dohmen W, Bonten MJ, Bos ME, et al. Carriage of extendedspectrum $\beta$-lactamases in pig farmers is associated with occurrence in pigs. Clin Microbiol Infect 2015;21:917-23.

28. Sun Q, Dyar OJ, Zhao L, et al. Overuse of antibiotics for the common cold - attitudes and behaviors among doctors in rural areas of Shandong Province, China. BMC Pharmacol Toxicol 2015;16:6:6.

29. Yu M, Zhao G, Stålsby Lundborg C, et al. Knowledge, attitudes, and practices of parents in rural China on the use of antibiotics in children: a cross-sectional study. BMC Infect Dis 2014;14:112.

30. Roschanski N, Fischer J, Guerra B, et al. Development of a multiplex real-time PCR for the rapid detection of the predominant beta-lactamase genes CTX-M, SHV, TEM and CIT-type AmpCs in Enterobacteriaceae. PLoS One 2014;9:e100956.

31. Pérez-Pérez FJ, Hanson ND. Detection of plasmid-mediated AmpC beta-lactamase genes in clinical isolates by using multiplex PCR. J Clin Microbiol 2002;40:2153-62.

32. Pasteran F, Tijet N, Melano RG, et al. Simplified protocol for carba NP Test for enhanced detection of carbapenemase producers directly from bacterial cultures. J Clin Microbiol 2015;53:3908-11.

33. Poirel L, Walsh TR, Cuvillier V, et al. Multiplex PCR for detection of acquired carbapenemase genes. Diagn Microbiol Infect Dis 2011;70:119-23.

34. Louie L, Goodfellow J, Mathieu P, et al. Rapid detection of methicillin-resistant staphylococci from blood culture bottles by using a multiplex PCR assay. J Clin Microbiol 2002;40:2786-90.

35. Stegger M, Andersen PS, Kearns A, et al. Rapid detection, differentiation and typing of methicillin-resistant Staphylococcus aureus harbouring either mecA or the new mecA homologue mecA(LGA251). Clin Microbiol Infect 2012;18:395-400.

36. The European committee on antimicrobial susceptibility testing EUCAST. http://www.eucast.org (accessed 18 May 2017)

37. The Clinical and Laboratory Standards Institute - CLSI. Global Standards for a Healthier World. http://clsi.org/ (accessed 18 May 2017).

38. ATC/DDD Index 2017. https://www.whocc.no/atc_ddd_index/ (accessed 18 May 2017).

39. Graneheim UH, Lundman B. Qualitative content analysis in nursing research: concepts, procedures and measures to achieve trustworthiness. Nurse Educ Today 2004;24:105-12.

40. Krippendorff K. Content analysis: an introduction to its methodology: SAGE Publications, Inc, 2012 